\title{
Nitrogen fertilizer affects the Populus microbiome
}

Emilie Sidelinger (Biosystems Engineering, Clemson University, Clemson, SC, USA) Eric Johnston (Biosciences Division, Oak Ridge National Laboratory, Oak Ridge, TN, USA) Dawn Klingeman (Biosciences Division, Oak Ridge National Laboratory, Oak Ridge, TN, USA) Melissa Cregger (Biosciences Division, Oak Ridge National Laboratory, Oak Ridge, TN, USA)

\begin{abstract}
Plants are colonized by numerous microorganisms serving important symbiotic functions that aid in plant growth and success. Populus deltoides (Eastern Cottonwood) and Populus trichocarpa (Black Cottonwood) are potential biofuel crops that rely on microbial symbionts to complete essential tasks, such as nitrogen fixation. Often, in agricultural and biofuel production, exogenous nitrogen is added to soils, since nitrogen availability is the limiting factor for plant growth. However, these additions may alter the soil microbial communities and result in unintentional consequences if excess nitrogen escapes the setting. We sought to determine how nitrogen fertilization alters the microbial communities within the soil, roots, and leaves of two cottonwoods. We grew $P$. deltoides and $P$. trichocarpa in a greenhouse with and without added nitrogen fertilizer, in two soil types. The soils were from Oregon and West Virginia and had distinctive chemical properties and different initial soil microbial communities. We hypothesized that the abundance
\end{abstract}


of diazotrophs within the soils would increase as nitrogen became limiting. Over the 2-month study, we assessed plant growth characteristics for over 160 Populus plants. We investigated the root, leaf, and soil microbiomes of selected plants using 16S rRNA gene and ITS2 PCR amplicon DNA sequencing. We then used bioinformatics tools to evaluate the composition and diversity of these microbiomes. The microbiomes differed significantly across all three experimental factors - nitrogen addition, Populus species, and soil type.

\section{BACKGROUND}

Plants and their microbiota have a dynamic relationship that benefits both biological groups. Recent advancements in our ability to investigate microbial communities has made more apparent the extent of this interdependence, which has in turn led to a shift in focus from the standalone plant model to ones that include the microorganisms and host plant as one entity, termed the "holobiont" (1). Such investigations often seek to identify roles that microorganisms have in plant health, survival, and productivity. Microorganisms have been shown to offer plant disease resistance (2) and stress tolerance (3). Even for anthropogenic uses, these microorganisms improve phytoremediation (4) and nutrient acquisition (5), leading to a higher biomass which benefits many industries, including organic farming.

Nitrogen is a limiting factor for plant growth. Most of terrestrial nitrogen is tied up as nitrogen gas. To enable nitrogen to be converted into a form that is available for both microorganisms and plants, microorganisms called diazotrophs expend energy to perform nitrogen fixation which converts plentiful dinitrogen gas into ammonium in the soil. Ammonium is converted to nitrate by another group of microorganisms which is also a plant-friendly form (6). A growing number of studies demonstrate that nitrogen is made available to the plant through 
associations with endophytic diazotrophs. However, a limited amount of studies have evaluated the effects of nitrogen fertilizer on the plant holobiont and the potential disruption of these key symbiotic associations.

Populus is a prime example of this trend- its holobiont has been defined, $(7,8,9)$ but no information has emerged on the effect of nitrogen fertilizer on the habitats within the metaorganism. Populus has recently gained attention, due to its potential as a biofuel feedstock. These plants have become a model organism for many reasons, including their classification as a short-rotation woody crop, and their ability to be hybridized within the genus to display a variety of desirable traits (10). Sequencing of the Populus trichocarpa genome (11) has allowed for additional genetic studies to differentiate between the Populus and its endophytic microorganisms in metagenomic studies.

The two Populus genotypes observed in this study include Populus trichocarpa (Black Cottonwood) and Populus deltoides (Eastern Cottonwood). These trees have been grown in either Oregon or West Virginia soils with different chemical compositions and microbial communities. Half of the subjects were fertilized with Hoagland's solution without nitrogen, and the other half with nitrogen. After 2-3 months of growth in a greenhouse, we characterized the microbial communities within the Populus roots and rhizospheres to identify shifts in the microbial communities due to nitrogen application. We hypothesized that nitrogen fertilizer application would impact microorganisms within the nitrogen cycle, specifically that the abundance of diazotrophs may decrease while microorganisms capable of denitrification may increase in relative abundance. 


\section{METHODS}

\section{A. Plant genotypes and greenhouse experiment}

The trees used in this study were Populus deltoides and P. trichocarpa, which were obtained from existing study locations Oregon and West Virginia. A total of 160 cuttings were acquired from live stake propagation and grown from May to July/August 2018 in a greenhouse located at Oak Ridge National Laboratory in Oak Ridge, TN, USA. Cuttings were grown in two varieties of soils from Oregon and West Virginia with contrasting parent material (table 1). Prior to experiment initiation, Oregon and West Virginia soils were mixed with sterile sand (50/50 mixture) to induce nitrogen-limiting conditions. During the course of the incubation, Hoagland's fertilizer (Basal mix \#2, Caisson Labs, Smithfield, UT, 84335) was applied weekly in $400 \mathrm{~mL}$ increments; half of these plants received Hoagland's fertilizer with nitrogen, while the other half received Hoagland's fertilizer without nitrogen.

Measurements of each trees' leaf number, height, diameter of stake cutting, diameter of growth off of stake, and average SPAD were taken at the initiation of experiment (May) and in July (2 months into experiment). At destructive sampling in July or August, aboveground and belowground wet biomass weight and a subset for dry biomass weight were recorded. Leaf, root, and rhizosphere samples were collected from all destructively sampled plants and stored at -80 degrees $\mathrm{C}$ for long term storage. $\mathrm{pH}, \mathrm{NH}_{4}-\mathrm{N}$, and $\mathrm{NO}_{3}-\mathrm{N}$ concentrations were measured for ten replicates from each experimental condition (i.e., genotype $\mathrm{x}$ soil type $\mathrm{x}$ fertilizer treatment) by the University of Georgia Agricultural \& Environmental Services Laboratories- Soil, Plant, and Water Laboratory. Basic soil chemistry was determined for triplicate samples of Oregon and West Virginia field soils, also by the University of Georgia Laboratory (table 1). 
Table 1 Chemical composition and characterization of field samples with no treatment. These values represent averages of triplicate samples.

\begin{tabular}{|c|c|c|c|c|c|c|c|c|c|c|c|c|}
\hline \multirow[b]{2}{*}{ Sample } & \multirow[b]{2}{*}{$\begin{array}{c}\mathrm{LBC}^{1} \\
\left(\mathrm{ppm}^{2}\right. \\
\mathrm{CaCO}_{3} / \\
\mathbf{p H})\end{array}$} & \multirow[b]{2}{*}{$\begin{array}{c}\mathbf{p H} \\
\mathrm{CaCl}_{2} \\
2\end{array}$} & \multirow[b]{2}{*}{$\begin{array}{c}\text { Equiv. } \\
\text { water } \\
\text { pH }\end{array}$} & \multicolumn{9}{|c|}{$\mathbf{m g} / \mathbf{k g}(\mathrm{ppm})$} \\
\hline & & & & $\mathbf{C a}$ & $\mathbf{K}$ & Mg & Mn & $\mathbf{P}$ & $\mathbf{Z n}$ & $\begin{array}{c}\mathrm{NH}_{4}- \\
\mathbf{N}\end{array}$ & $\begin{array}{c}\mathrm{NO}_{2-}- \\
\mathbf{N}\end{array}$ & $\begin{array}{c}\mathbf{N O}_{3-} \\
\mathbf{N}\end{array}$ \\
\hline $\begin{array}{c}\text { West } \\
\text { Virginia } \\
\end{array}$ & 462 & 5.50 & 6.10 & 1268 & 169.8 & 177.3 & 22.68 & 5.64 & 2.75 & 7.70 & 0.25 & 19.65 \\
\hline Oregon & 1005 & 4.87 & 5.47 & 2240 & 169.5 & 351.2 & 19.75 & 33.20 & 5.60 & 7.32 & 0.27 & 20.95 \\
\hline
\end{tabular}

B. Sample processing- DNA extraction

Soil DNA was extracted following the DNeasy PowerSoil Pro Kit (Qiagen, Venlo, The Netherlands), using two subsamples of $0.25 \mathrm{~g}$ for duplicate extractions, which were subsequently combined downstream. To remove residual impurities in DNA extracts, these samples were cleaned using Agencourt Ampure XP beads (Beckman Coulter Inc., Pasadena, CA, USA). Roots were surface sterilized using three 30 second water wash cycles, followed by a 30 second wash of $70 \% \mathrm{EtOH}$, and another set of three 30 second water wash cycle. Sodium hypochlorite was not used because it was found to eliminate all DNA from a batch of test samples. After washing, the fine roots were thinly sliced with a razor blade prior to DNA extraction. These fine roots were then bead beaten in liquid nitrogen frozen blocks using a TissueLyser II (Qiagen, Venlo, The Netherlands) at $30 \mathrm{rpm}$ for 3 minutes with a 5-mm steel bead to pulverize the sample. Afterwards, Qiagen DNeasy PowerPlant Pro Kit (Qiagen, Venlo, the Netherlands) was used for DNA isolation, followed by a Qiagen (formerly MoBio) DNA cleanup kit and Agencourt AMPure XP beads to remove any possible contaminants. Because leaves had been frozen prior to extraction, whole leaf extraction was performed to analyze both the leaf phylloplane and endosphere jointly. These 
samples were bead beaten in liquid nitrogen frozen blocks for 1 minute also using a TissueLyser II at $30 \mathrm{rpm}$. The pulverized samples then underwent extraction using a Qiagen DNeasy PowerPlant Pro Kit, along with a DNeasy PowerClean CleanUp Kit and Ampure beads. Post extraction, all samples (i.e., soil, root, and leaf) were quantified using a Nanodrop 1000 spectrophotometer (NanoDrop Products, Wilmington, DE, USA) and Qubit 2.0 fluorometer (Thermo Fisher Scientific, Waltham, MA, USA).

\section{Amplicon library preparation}

In this project we used amplicon sequencing to investigate microbial diversity in soil and root samples. Amplicons were prepared following Illumina's protocol for 16s Metagenomic Sequencing Library preparation (Part\# 15044223 RevB, Illumina Inc. Hayward CA). In our study we used the following primers 515F, 515F_f1C, 515F_f1TM7, 515F_f4Arc (forward) and 806R (reverse) (Lane et al. 1985 and Shakya et al. 2013) for 16s (fig. 1). Primers were designed according to the Illumina protocol including an Illumina overhang. In short, amplicons were amplified by polymerase chain reaction (PCR) individually with the 16s rRNA primers. The amplified products were visualized by gel electrophoresis, and subsequently cleaned using SPRI magnetic beads (Beckman Coulter, Indianapolis, USA). The cleaned product was then used as template in limited cycle (8) PCR reaction to add unique indexes to each sample. Once the indexes were added the products were cleaned with SPRI magnetic beads. The final indexed samples were then quantified using a Nanodrop 1000 instrument (ThermoScientific, Waltham, MA), and samples were pooled ( $\sim 8$ samples per pool) in an equal Molar fashion. Pools were assessed on a Bioanalyzer DNA 7500 DNA Chip (Agilent, Santa Clara, CA, USA), and then combined into a single final pool. The final pool was quantified using a Qubit fluorometer (Life 
Technologies, Carlsbad, CA, USA). Pooled indexed libraries were diluted according to the manufactures recommendations and sequenced with a PhiX spike in (10-20\%) in two directions 251 bases (PE251) on an Illumina MiSeq instrument (Illumina Inc. San Diego, CA). Samples were de-multiplexed as part of the sequencing process, and then analyzed.

\begin{tabular}{lllll}
\hline $\begin{array}{l}\text { Primer } \\
\text { name }\end{array}$ & Sequence & Direction & Target & Reference \\
\hline $515 \mathrm{~F}$ & $\begin{array}{l}\text { TCGTCGGCAGCGTCAGATGTGTATAAGA } \\
\text { GACAGGTGCCAGCMGCCGCGGTAA }\end{array}$ & Forward & $\begin{array}{l}\text { Bacteria/ } \\
\text { Archaea }\end{array}$ & $\begin{array}{l}\text { Lane et al. } \\
1985\end{array}$ \\
\hline $515 \mathrm{~F}_{f}$ f1C & $\begin{array}{l}\text { TCGTCGGCAGCGTCAGATGTGTATAAGA } \\
\text { GACAGGTGCCAGCMGCWGCGGTAA }\end{array}$ & Forward & Cloroflexi & $\begin{array}{l}\text { Shakya et } \\
\text { al. 2013 }\end{array}$ \\
\hline $515 \mathrm{~F}_{-}$f1TM7 7 & $\begin{array}{l}\text { TCGTCGGCAGCGTCAGATGTGTATAAGA } \\
\text { GACAGGTGCCAGCMGCCGCGGTCA }\end{array}$ & Forward & TM7 & $\begin{array}{l}\text { Shakya et } \\
\text { al. 2013 }\end{array}$ \\
\hline $515 F_{-}$f4Arc & $\begin{array}{l}\text { TCGTCGGCAGCGTCAGATGTGTATAAGA } \\
\text { GACAGGTGKCAGCMGCCGCGGTAA }\end{array}$ & Forward & Archaea & $\begin{array}{l}\text { Shakya et } \\
\text { al. 2013 }\end{array}$ \\
\hline 806R & $\begin{array}{l}\text { GTCTCGTGGGCTCGGAGATGTGTATAAG } \\
\text { AGACAGGGACTACHVGGGTWTCTAAT }\end{array}$ & Reverse & $\begin{array}{l}\text { Bacteria/ } \\
\text { Archaea }\end{array}$ & $\begin{array}{l}\text { Lane et al. } \\
1985\end{array}$ \\
\hline
\end{tabular}

Fig. 1 PCR primers used to amplify hypervariable regions within 16s gene among Bacteria and Archaea. Black signifies the Illumina adaptor; red signifies the primer sequence.

D. Computational analysis

Using BBmerge (part of the BBtools package) the paired-end DNA sequences were merged bioinformatically (12). Primer sequences used in PCR were removed from the forward and reverse strands, and sequences were trimmed based on a quality score of 17 and a window across an averaging window of 3 base pairs (bp). Sequences less than $150 \mathrm{bp}$ were discarded. Following merging and trimming, DADA2 within the QIIME2 software package was used to denoise and dereplicate sequences into sequence variants (13). We used the QIIME2 feature classifier to organize our sequences into their taxonomic classifications, and community composition was then summarized at the phylum level (14). Mitochondria and chloroplast sequences (from host DNA) were removed from root datasets, which typically represented $55-80 \%$ of community datasets. 
Differences in the relative abundance of microbial phyla between $+\mathrm{N} /-\mathrm{N}$ groups were evaluated using unpaired student's t-test. Beta-diversity was calculated using the Yule distance method.

\section{RESULTS AND DISCUSSION}

\section{A. Greenhouse measurements}

After being fertilized separately, the $+\mathrm{N}$ soils differentiated itself from the $-\mathrm{N}$ control groups. This characteristic is especially true of nitrate composition (fig. 2). The -N groups' nitrate concentration remains consistent across genotype and soil origin. Although the $+\mathrm{N}$ groups were given the same nitrogen application schedule, the ending soil nitrate concentrations have much more variability, especially between the Oregon $P$. deltoides and $P$. trichocarpa groups. This difference in concentrations may be due to excess nitrogen runoff, uptake by the plant, or microbial intervention.

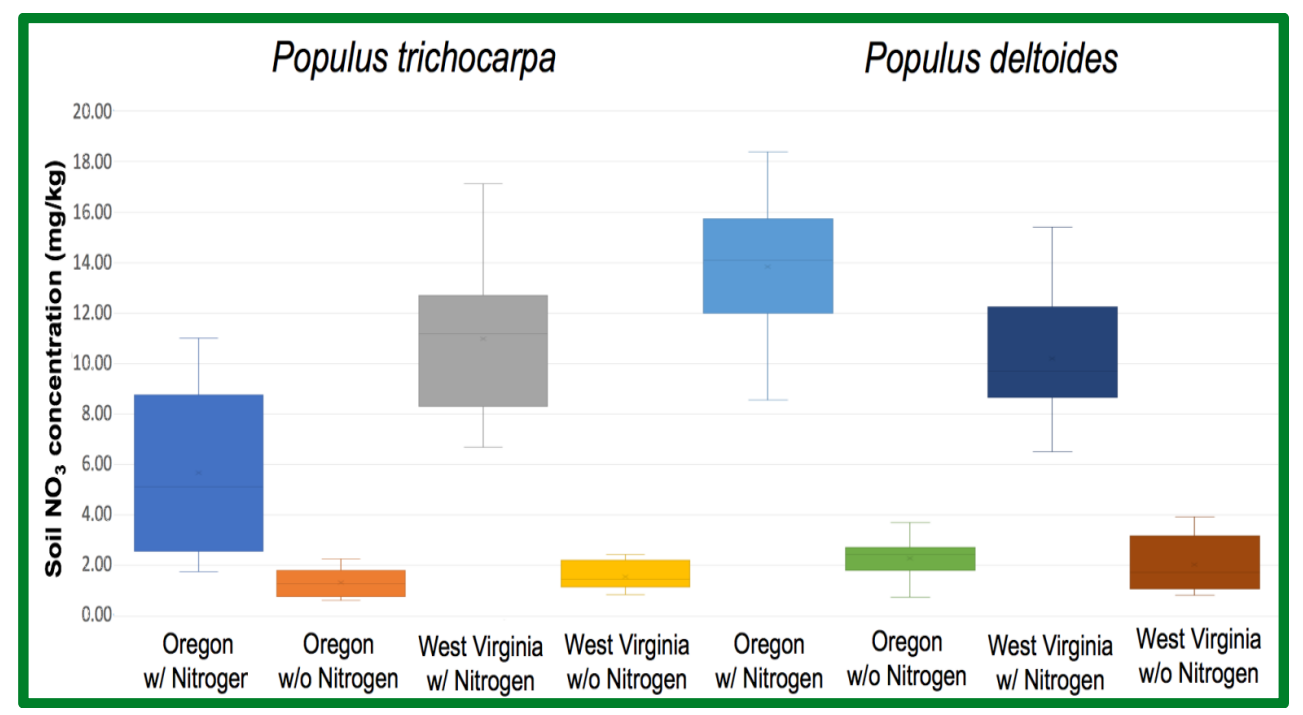

Fig. 2 Final soil nitrate composition shows consistency within $-\mathrm{N}$ and variability in $+\mathrm{N}$ groups. 
For both genotypes and soil types, Populus subjects that had been fertilized with nitrogen demonstrated a significantly greater amount of growth over the 2-3 month test period. $P$. trichocarpa grew more by a factor of 4.5, and P. deltoides by a factor of 2.8 (fig. 3). These results also demonstrate the degree of nitrogen limitation resulting from the sand-soil mixture.

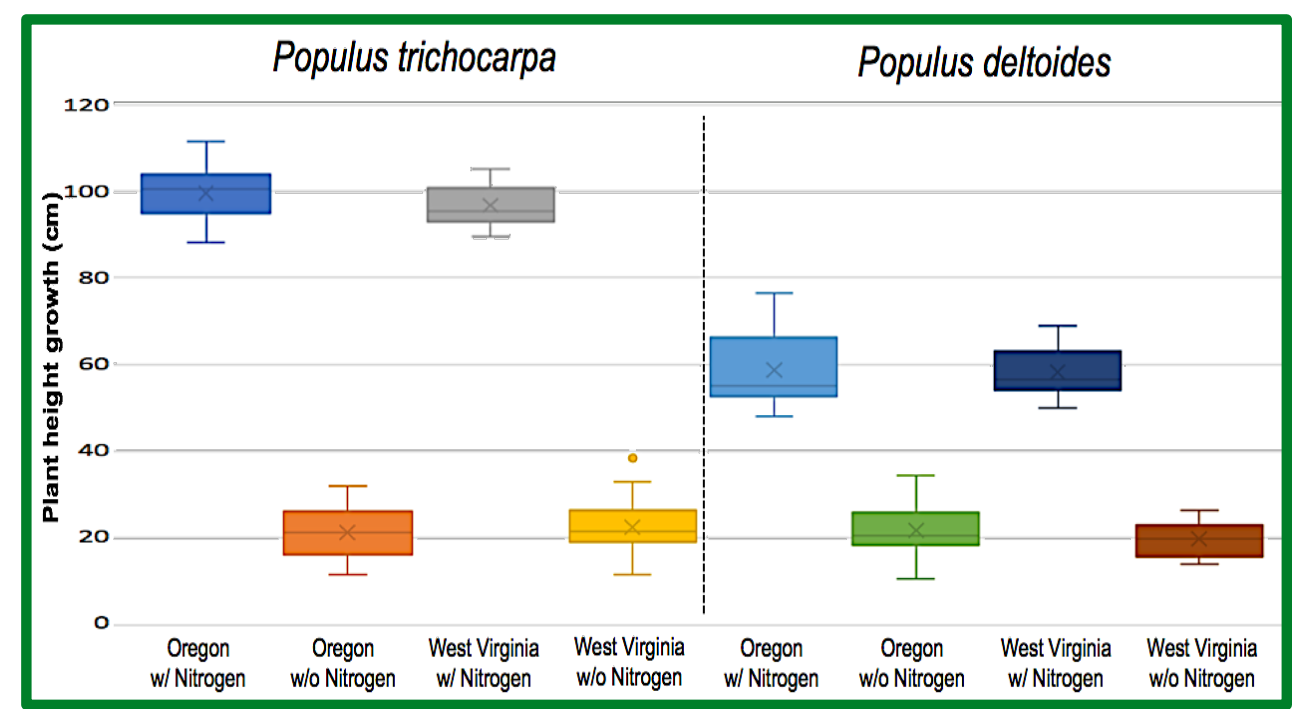

Fig. 3 Plant height after a 2-3 month growth period under variable conditions. These heights reflect growth incurred while under the test conditions.

While nitrogen was found to support new growth, excess nitrogen was also shown to be allocated to the existing structure, including the leaves (fig 4). This increase in nitrogen may go towards building proteins and further promoting plant health. 


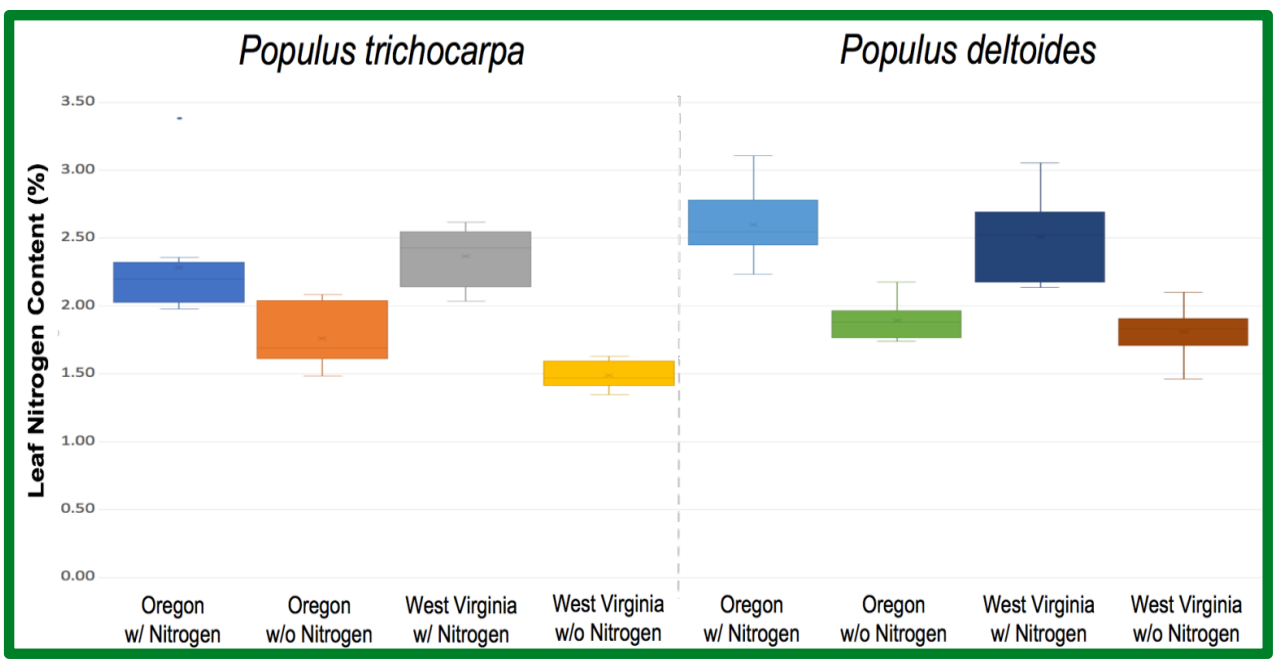

Fig. 4 Percentage of nitrogen within leaf samples.

The change in average SPAD also continued this trend, with a higher chlorophyll density in trees fertilized with nitrogen (fig. 5). These results seem to also be dependent on genotype, as the average SPAD within the $P$. deltoides subjects mostly decreased while average SPAD within P. trichocarpa often increased.



Fig. 5 Change in average SPAD over a 2 month growth period. 
B. Root and rhizosphere microbial communities

When shifted from the field setting to the rhizosphere of the Populus, the field microbial composition reveals a statistically significant $(\mathrm{P}$-value $<0.05)$ increase in Proteobacteria and Armatimonadetes with a decrease in Actinobacteria and Bacteroidetes (Figure 2). Many species within Proteobacteria are known to be involved in nitrogen fixation (25), which may have been affected by nitrogen addition.

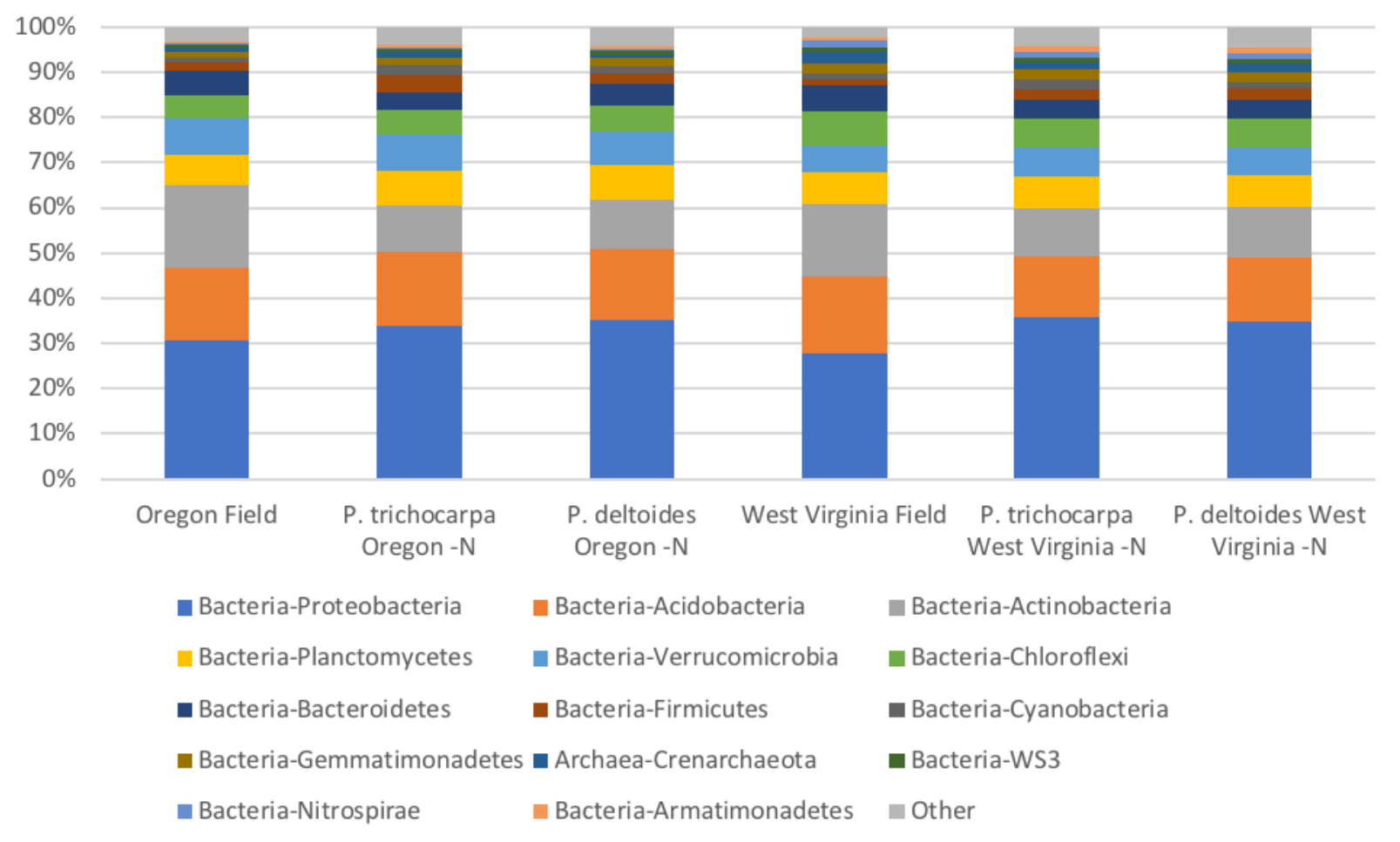

Fig. 6 Field and rhizosphere soils differ in microbial composition

A t-test revealed that the microbial communities between the $-\mathrm{N} /+\mathrm{N}$ groups had no consistent difference among variables, besides that of the FCPU426 population, which accounted for a small fraction $(.1 \%-.3 \%)$ of the community. While only a few species were shown to have significant differences in amount between the $-\mathrm{N} /+\mathrm{N}$ groups of each condition, these amount 
differences were drastic. For example, the $P$. deltoides Oregon root population of

Verrucomicrobia differed from $6 \%$ in the $-\mathrm{N}$ setting and $21 \%$ in the $+\mathrm{N}$ setting (fig. 7 ).

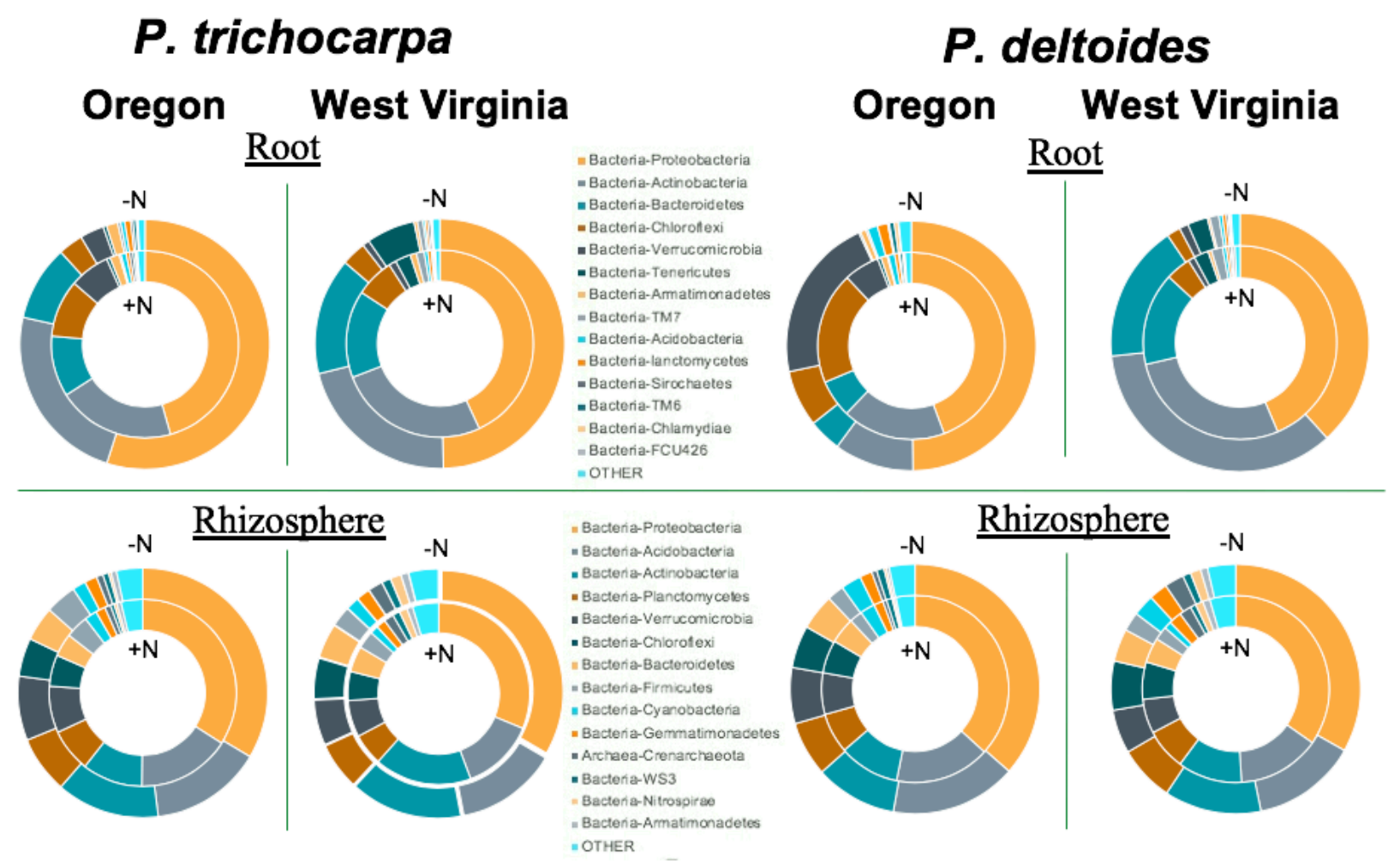

Fig. 7 Phyla variations in root and rhizosphere microbial communities

The PCoA plot using the Yule distance demonstrated that soil microbial communities from each experimental group tend to form distinct clusters (fig. 8). The difference in these communities is especially apparent between the two different field soils used in the experiment. 


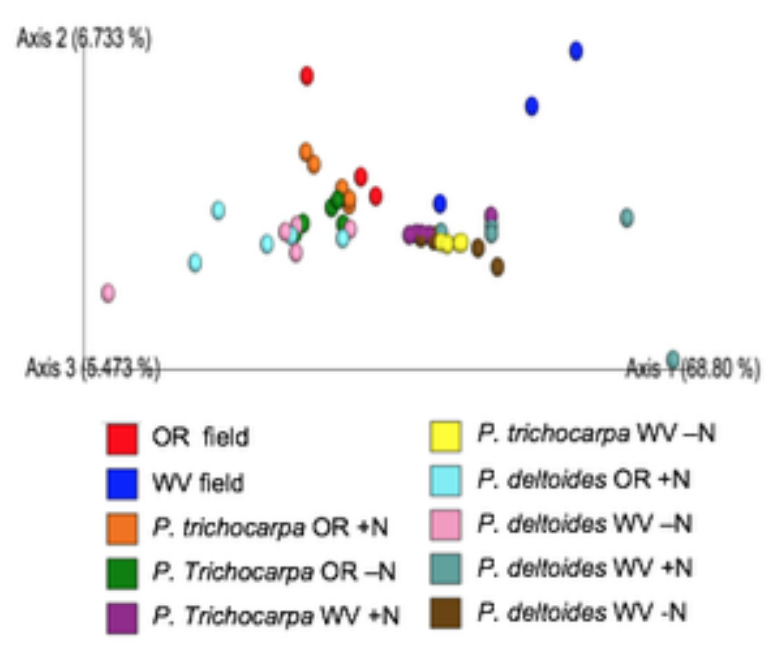

Fig. 8 Beta diversity PCoA plot using Yule distance calculation method for rhizosphere communities

\section{CONCLUSIONS}

This study has begun to demonstrate the impact of nitrogen fertilizer on the Populus holobiont. While some initial correlations were shown, there are many aspects of the soil and root microbial communities we have yet to explore. Conclusive results would rely on further breaking down the taxonomy, to see the effect of nitrogen addition on the microorganisms that facilitate the nitrogen cycle. Also, analysis of fungal rhizobia and endophytes will be needed to holistically demonstrate the microbe-plant interaction under these conditions. Analyzing additional niches belonging to the plant, such as the leaves, may also be necessary to draw conclusive results.

\section{ACKNOWLEDGEMENTS}

This work was supported in part by the U.S. Department of Energy, Office of Science, Office of Workforce Development for Teachers and Scientists (WDTS) under the Science Undergraduate Laboratory Internships (SULI) program at Oak Ridge National Laboratory (ORNL), administered by the Oak Ridge Institute for Science and Education. This study was also supported and 
performed as part of the Center for BioEnergy Innovation (CBI) at ORNL. CBI is a U.S.

Department of Energy Bioenergy Research Center supported by the Office of Biological and Environmental Research in the DOE Office of Science.

\section{REFERENCES}

1. Zilber-Rosenberg I, Rosenberg E (2008) Role of microorganisms in the evolution of animals and plants: the hologenome theory of evolution. FEMS microbiology reviews 32(5):723-735.

2. Berendsen RL, Pieterse CMJ, Bakker PAHM (2012) The rhizosphere microbiome and plant health. Trends in plant science 17(8):478-486.

3. Doty SL (2011) Nitrogen-Fixing Endophytic Bacteria for Improved Plant Growth (SPRINGER-VERLAG BERLIN, BERLIN; HEIDELBERGER PLATZ 3, D-14197 BERLIN, GERMANY) doi:10.1007/978-3-642-20332-9_9.

4. Thijs S, Sillen W, Rineau F, Weyens N, Vangronsveld J (2016) Towards an Enhanced Understanding of Plant-Microbiome Interactions to Improve Phytoremediation: Engineering the Metaorganism. Frontiers in Microbiology 7:341.

5. Pii Y, et al. (2015) Microbial interactions in the rhizosphere: beneficial influences of plant growth-promoting rhizobacteria on nutrient acquisition process. A review. Biology and Fertility of Soils 51(4):403-415.

6. Santi C, Bogusz D, Franche C (2013) Biological nitrogen fixation in non-legume plants. Annals of Botany 111(5):743-767.

7. Cregger MA, et al. (2018) The Populus holobiont: dissecting the effects of plant niches and genotype on the microbiome. Microbiome 6:31.

8. Shakya M, et al. (2013) A Multifactor Analysis of Fungal and Bacterial Community Structure in the Root Microbiome of Mature Populus deltoides Trees. Plos One 8(10): 76382.

9. Gottel NR, et al. (2011) Distinct Microbial Communities within the Endosphere and Rhizosphere of Populus deltoides Roots across Contrasting Soil Types. Applied and Environmental Microbiology 77(17):5934-5944.

10. Sannigrahi P, Ragauskas AJ, Tuskan GA (2010) Poplar as a feedstock for biofuels: A review of compositional characteristics. Biofuels Bioproducts \& Biorefining-Biofpr 4(2):209-226.

11. Tuskan GA, et al. (2006) The genome of black cottonwood, Populus trichocarpa (Torr. \& Gray). Science 313(5793):1596-1604. 
12. Bushnell B, Rood J, Singer E (2017) BBMerge - Accurate paired shotgun read merging via overlap. Plos One 12(10):e0185056.

13. Callahan BJ, et al. (2016) DADA2: High-resolution sample inference from Illumina amplicon data. Nature Methods 13(7):+.

14. Caporaso JG, et al. (2010) QIIME allows analysis of high-throughput community sequencing data. Nature Methods 7(5):335-336. 